\title{
Protocadherin-1: epithelial barrier dysfunction in asthma and eczema
}

\author{
Grissel Faura Tellez $z^{1,2,3,4}$, Martijn C. Nawijn $n^{2,3}$ and Gerard H. Koppelman ${ }^{1,3}$
}

Affiliations: 'University of Groningen, University Medical Center Groningen, Beatrix Children's Hospital, Dept of Pediatric Pulmonology and Pediatric Allergology, Groningen, ${ }^{2}$ University of Groningen, University Medical Center Groningen, Laboratory of Allergology and Pulmonary Diseases, Dept of Pathology and Medical Biology, Groningen, and ${ }^{3} \mathrm{GRIAC}$ Research Institute, University of Groningen, University Medical Center Groningen, Groningen, The Netherlands. ${ }^{4} \mathrm{Clinical}$ and Experimental Sciences, Faculty of Medicine, University of Southampton, Southampton, UK.

Correspondence: G.H. Koppelman, Pediatric Pulmonology and Pediatric Allergology, Beatrix Children's Hospital, University Medical Center Groningen, PO Box 30.001, 9700 RB Groningen, The Netherlands. E-mail: g.h.koppelmanđuumcg.nl

0 @ERSpublications A role for protocadherin-1 in loss of epithelial integrity in both asthma and eczema http://ow.ly/qM9mE

Asthma and eczema result from the interaction between genetic susceptibility and environmental exposures. Over the last two decades, genetic studies have led to the identification of multiple susceptibility genes, increasing our understanding of the biological pathways leading to these diseases.

Many asthma and eczema susceptibility genes are expressed in epithelial cells of airway mucosa and skin [1]. The epithelium constitutes the first line of defence against allergens and invading microbes. Recently, loss of epithelial integrity has been implicated as an important mechanism leading to both asthma [2] and eczema [3].

In this issue of the European Respiratory Journal, MORTENSEN et al. [4] report the association of genetic variants in the gene encoding protocadherin-1 ( $P C D H 1)$ with asthma, wheeze and eczema in (early) childhood in the Danish birth cohort study COPSAC (Copenhagen Studies on Asthma in Childhood). Their studies also suggest a gene-environment interaction of $P C D H 1$ with passive smoke exposure in early childhood in asthma development. These studies expand on previous data reporting a role for $\mathrm{PCDH} 1$ polymorphisms in the susceptibility to both asthma [4-7] and eczema $[4,6,8]$. Given the replicated association of $P C D H 1$ with both diseases, this gene might contribute to a biological pathway that constitutes a shared cause for both diseases. Here, we will summarise the cumulative data on $P C D H 1$ polymorphisms in asthma and eczema, and make an attempt to interpret these genetic data in light of PCDH1 expression and function as a contributor to epithelial integrity.

In 1993, PCDH1 was originally identified by SANO et al. [9] as protocadherin-42 and was reported to induce cell adhesion upon ectopic membrane expression in a mouse fibroblast L-cell assay. PCDH1 is a member of the $\delta 1$ subfamily of the nonclustered protocadherin genes. PCDH1 is characterised by seven extracellular cadherin (EC) repeats, a transmembrane domain and three evolutionarily conserved motifs (CM1-3) in the intracellular tail that have been suggested to participate in intracellular signalling processes [10]. The $P C D H 1$ gene spans $25 \mathrm{~kb}$ on chromosome 5q31-33 and encodes two main transcripts that contain either three or five exons through alternative splicing. The three-exon isoform does not encode the cytoplasmic

Received: Oct 152013 | Accepted: Oct 172013

Support statement: G.F. Tellez is supported by a grant from the University Medical Center Groningen and the University of Southampton. The authors were supported by the Netherlands Asthma Foundation grant 3.2.09.055 and MEDALL, a collaborative project supported by the European Union under the Health Cooperation Work Programme of the 7th Framework programme (grant agreement number 261357).

Conflict of interest: Disclosures can be found alongside the online version of this article at www.erj.ersjournals.com 
domains CM1-3 that are present in the five-exon isoform. Recently, a sixth exon and alternative expression of exon 1 were identified in bronchial epithelial cells [11], whereas skin epithelial expression of PCDH1 has not been extensively characterised to date.

In 2009, PCDH1 was identified as a susceptibility gene for bronchial hyperresponsiveness (BHR) and asthma in children and adults. Subsequently, PCDH1 was shown to be expressed in epithelial cells of the airway [5,9] and the skin [12]. In four genetic association studies on PCDH1 published to date, PCDH1 gene variants were associated with BHR, asthma, eczema, nonallergic childhood asthma and transient early wheeze (table 1) [4-6, 8]. However, in large-scale genome-wide association studies of asthma, such as the GABRIEL consortium study [7], $\mathrm{PCDH} 1$ was not reported to be associated, at a genome-wide significance level, with asthma defined as a self-reported "asthma ever" diagnosis. In our opinion, these contradictory results may be explained by asthma heterogeneity.

Asthma is a chronic heterogeneous disease of the airways with different disease subtypes, characterised by specific onset of respiratory symptoms, such as cough and wheeze. MORTENSEN et al. [4] report, in a longitudinal analysis of specific disease subtypes, that $P C D H 1$ gene variants were associated with specific temporal episodes of wheezing and coughing symptoms, and with the onset of asthma. Moreover, characteristic respiratory symptom patterns in early childhood, such as transient early respiratory symptoms and recurrent troublesome symptoms, were associated with different $P C D H 1$ polymorphisms. These specific associations with subphenotypes of the disease may well have been missed in an association analysis with "Have you ever had asthma?" as an outcome parameter.

In summary, while different association analyses identify a variety of genetic signals in the $P C D H 1$ gene, the general picture that starts to emerge indicates that $P C D H 1$ gene function is associated with asthma, BHR and eczema in early life. Importantly, gene by passive smoking interactions were found to be relevant for the association of $P C D H 1$ with asthma in two populations $[4,5]$. These data suggest that the contribution of

TABLE 1 Associations of PCDH1 SNPs with asthma and eczema

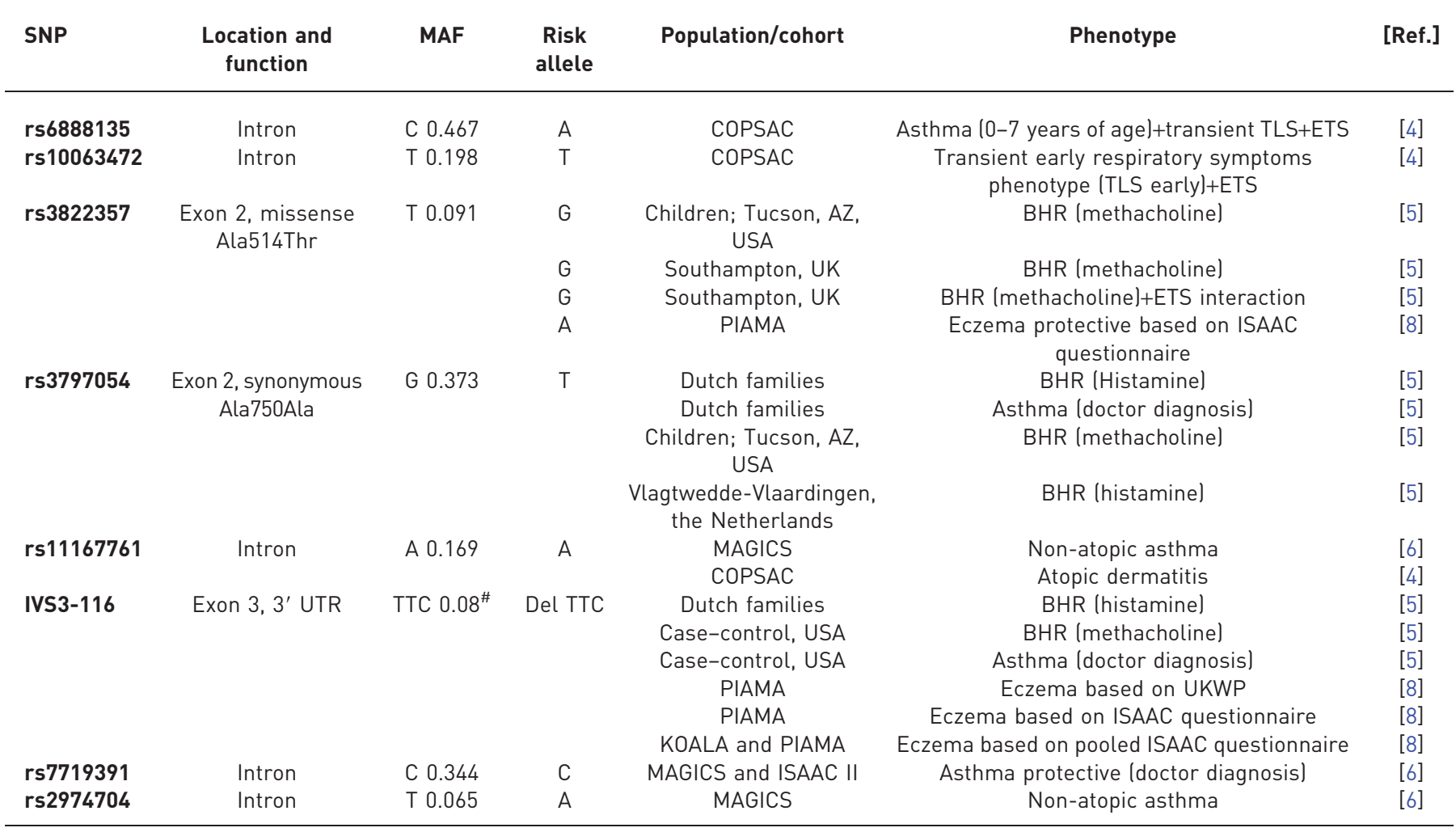

PCDH1: protocadherin-1; SNP: single-nucleotide polymorphism; MAF: minor allele frequency; rs: reference SNP; COPSAC: Copenhagen Studies on Asthma in Childhood; TLS: troublesome lung symptoms; ETS: environmental tobacco smoke; BHR: bronchial hyperresponsiveness; MAGICS: Multicentre Asthma Genetics in Childhood Study; PIAMA: Prevention and Incidence of Asthma and Mite Allergy; UKWP: UK Working Party; ISAAC: International Study of Asthma and Allergies in Childhood; Del: deletion. MAF was obtained from dbSNP. Risk allele tested for association varied in different cohorts. ${ }^{\#}$ : only in Dutch population. 


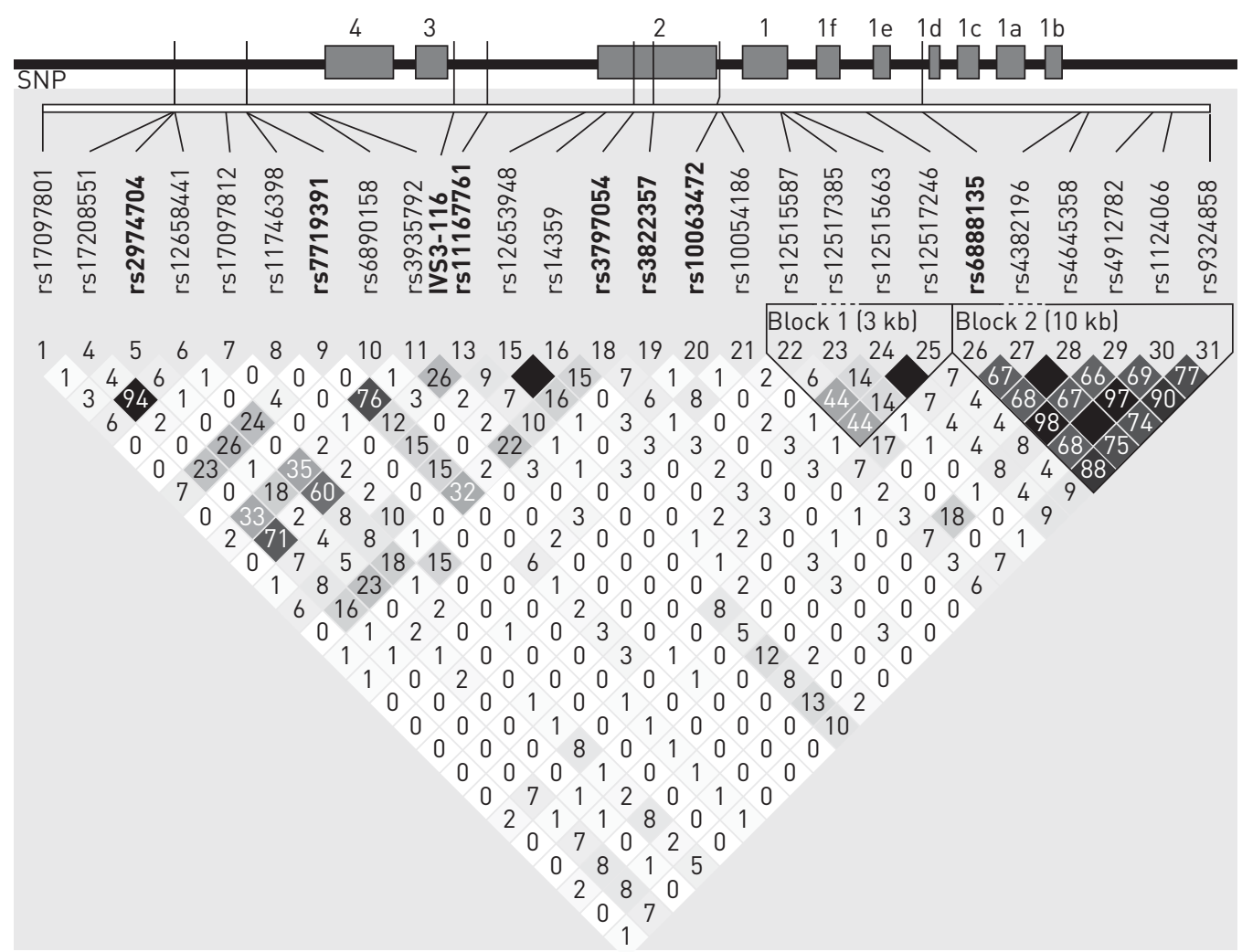

FIGURE 1 Protocadherin-1 gene, single-nucleotide polymorphism (SNP) positions and linkage disequilibrium (LD) pattern: position on reference contig NT_ AC094107.3 (Homo sapiens) based on genomic data. LD plots are displayed in $r^{2}$ values. The stronger the LD, the darker the squares; $r^{2}=0$ is white and $r^{2}=1$ is represented in black. LD plots were constructed with Haploview (version 4.2) software using SNP data from the HapMap Project (http://hapmap.ncbi.nlm. nih.gov/) for the CEU population. Bold type represents SNPs that also appear in table 1.

PCDH1 gene variants to asthma may become evident when studied in the right environmental context. Well-powered, collaborative meta-analyses of cohorts with specific asthma subphenotypes in early life are needed to provide more robust evidence of these associations (table 1). Finally, the lack of association with specific or total IgE levels or with allergic asthma [6] suggests that PCDH1 is relevant to non-IgE-mediated mechanisms of disease susceptibility. Consequently, the contribution of $P C D H 1$ to asthma susceptibility appears to be IgE independent (table 1).

The cumulative data gathered to date indicate that PCDH1 harbours a complexity of genetic signals associated with asthma and eczema that we do not yet fully understand, without a clear dominant polymorphism that is associated with asthma or eczema in most studies. In order to understand the interrelation of these associations observed for the different PCDH1 single-nucleotide polymorphisms (SNPs), we considered the possibility of linkage disequilibrium (LD) between these SNPs. LD is defined as the preferential occurrence of specific combinations of SNPs in the population, due to their co-inheritance as a result of their close physical proximity. In a recent study in a German population, Toncheva et al. [6] investigated the possibility that associations of $P C D H 1$ with asthma were caused by LD with the nearby cytokine cluster on chromosome 5q31-33, but this was not the case. Our analysis of the LD pattern in Caucasian subjects did not reveal strong evidence for LD between the various SNPs (fig. 1), indicating that these SNPs do not represent a single genetic signal but apparently make individual, independent contributions to disease susceptibility.

Another explanation for the identification of multiple PCDH1 SNPs to be independently associated with asthma and eczema in different studies is the presence of multiple SNPs within the PCDH1 gene that have diverse functional effects. Firstly, very specific SNPs might interact with environmental factors such as passive smoke exposure. Secondly, other SNPs may have more generic effects, for instance, by regulating PCDH1 gene expression. It is important to realise that the SNPs tested in the original study by KopPELMAN et al. [5] were only situated in coding regions of the gene. Over the past years, it has become increasingly clear that noncoding SNPs can have important regulatory effects on gene expression (by acting as 
expression quantitative trait loci (eQTLs)). The current publication by MORTENSEN et al. [4] adds the association of further noncoding $P C D H 1$ polymorphisms to asthma and eczema (table 1), whereas none of the initial associations were replicated in this study. Thus, eQTL studies on these PCDH1 SNPs in lung and skin tissue are therefore urgently needed to assess their functional relevance. Thirdly, coding SNPs may have generic effects by affecting PCDH1 protein function. The coding SNP Ala514Thr maps to an EC domain in the PCDH1 protein, which is compatible with a potential effect on cell-cell adhesion that needs further study. So far, no data are available on the functional effects of any of the asthma- and eczema-associated SNPs. Finally, adding to the complexity of $P C D H 1$, its expression is also probably regulated by epigenetic mechanisms that include gene methylation, given the identification of exon $1 \mathrm{~A}$ as a CpG island that is potentially methylated [11].

So, how does PCDH1 contribute to both asthma and eczema? We hypothesise that PCDH1 dysfunction contributes to the defect in epithelial barrier function observed in both asthma and eczema by regulating the process of epithelial cell differentiation and/or repair. PCDH1 mRNA and protein expression is strikingly upregulated during primary bronchial epithelial cell differentiation, suggesting a role for PCDH1 in achieving or maintaining terminal differentiation of the airway epithelium [11]. The regulation of PCDH1 expression in the skin during epidermal differentiation has not been characterised to date, although PCDH1 expression is markedly induced in primary keratinocytes late during the repair process after scratch wounding [12]. A similar role in epidermal differentiation has been previously identified for multiple eczema genes in genetic studies. All in all, we propose a role for PCDH1 in epithelial differentiation and repair in both asthma and eczema.

PCDH1 is capable of intracellular signalling. Downstream pathways or PCDH1 interaction partners relevant to asthma or eczema pathogenesis have not been reported to date. Moreover, data on the differential expression of PCDH1 in asthma or eczema versus healthy controls are still lacking. Future studies are needed that can validate the functional role of PCDH1 in asthma and eczema by controlled experimental approaches, such as small interfering RNA or genetically engineered mouse models, that are currently being developed. These studies also need to address the possibility of environmental exposures affecting PCDH1 expression, with cigarette smoke exposure being of particular interest. Clearly, more studies are needed to resolve the functional effects of $P C D H 1$ SNPs and their relevance for airway and skin epithelial cell biology. These studies will ultimately validate the initial genetic findings and perhaps provide clues for future novel therapeutic interventions in asthma and eczema.

\section{References}

Lambrecht BN, Hammad H. Death at the airway epithelium in asthma. Cell Res 2013; 23: 588-589.

Xiao C, Puddicombe SM, Field S, et al. Defective epithelial barrier function in asthma. J Allergy Clin Immunol 2011; 128: $549-556$

Hon KL, Leung AK, Barankin B. Barrier repair therapy in atopic dermatitis: an overview. Am J Clin Dermatol 2013; 14: 389-399.

4 Mortensen LJ, Kreiner-Møller E, Hakonarson H, et al. The PCDH1 gene and asthma in early childhood. Eur Respir J 2014; 43: 792-800.

5 Koppelman GH, Meyers DA, Howard TD, et al. Identification of PCDH1 as a novel susceptibility gene for bronchial hyperresponsiveness. Am J Respir Crit Care Med 2009; 180: 929-935.

6 Toncheva AA, Suttner K, Michel S, et al. Genetic variants in Protocadherin-1, bronchial hyper-responsiveness, and asthma subphenotypes in German children. Pediatr Allergy Immunol 2012; 23: 636-641.

7 Moffatt MF, Gut IG, Demenais F, et al. A large-scale, consortium-based genomewide association study of asthma N Engl J Med 2010; 363: 1211-1221.

8 Koning H, Postma DS, Brunekreef B, et al. Protocadherin-1 polymorphisms are associated with eczema in two Dutch birth cohorts. Pediatr Allergy Immunol 2012; 23: 270-277.

9 Sano K, Tanihara H, Heimark RL, et al. Protocadherins: a large family of cadherin-related molecules in central nervous system. EMBO J 1993; 12: 2249-2256.

10 Redies C, Vanhalst K, Roy F. $\delta$-Protocadherins: unique structures and functions. Cell Mol Life Sci 2005; 62: 2840-2852.

11 Koning H, Sayers I, Stewart CE, et al. Characterization of protocadherin-1 expression in primary bronchial epithelial cells: association with epithelial cell differentiation. FASEB J 2012; 26: 439-448.

12 Fitsialos G, Chassot AA, Turchi L, et al. Transcriptional signature of epidermal keratinocytes subjected to in vitro scratch wounding reveals selective roles for ERK1/2, p38, and phosphatidylinositol 3-kinase signaling pathways. J Biol Chem 2007; 282: 15090-15102. 